\title{
Métodos para aplicar las pruebas de aceptación para la alimentación escolar: validación de la tarjeta lúdica
}

\author{
Methods for applying the tests of \\ acceptability for school feeding: \\ the validation of playful cards
}

\begin{abstract}
The objective of this study was to validate methods to assess the acceptability on scholar feeding. A total of 354 students took part in this survey, from second to fifth years of public education system in Santos city, São Paulo, Brazil. A comparison between three methods was used: mixed facial hedonic scale of five points, playful cards and leftover analysis that was the reference method. Kappa statistics and quadratic kappa were used to verify the concordance between the methods. There was an estimated reasonable agreement between the leftover analysis and the affective methods: mixed facial hedonic scale (kappa $=0.2414)$ and playful cards (kappa=0.2758), all of them with $p<0,001$. When compared the mixed facial hedonic scale and the playful cards, it was observed a quadratic kappa of 0.3846. It is recommended that the application of the validated methods in the school context be studied in different spheres of government and in other countries as well.

Key-words: School feeding; validations studies; students; feeding.
\end{abstract}

\section{INTRODUCCIÓN}

El Programa Nacional de Alimentación Escolar (PNAE) es el programa brasileño que tiene como objetivo satisfacer las necesidades nutricionales de los estudiantes durante su estancia en el aula, favoreciendo el crecimiento, desarrollo, aprendizaje, rendimiento escolar y la formación de hábitos alimentarios saludables. En este contexto, el programa proporciona comidas gratuitas a todos los estudiantes de educación básica en escuelas públicas y filantrópicas que incluyen: centros de educación preescolar, guardería infantil, escuelas primarias, escuelas secundarias y de jóvenes y adultos $(1,2)$.

PNAE se caracteriza principalmente por su universalidad, equidad y la operación descentralizada. Por estas razones se considera ejemplo en la gestión de los programas de alimentación escolar en el extranjero.

El año 2007, Brasil comenzó un trabajo conjunto con el Programa Mundial de Alimentos (PMA) de la Organización de las Naciones Unidas para la Agricultura y la Alimentación (FAO) en la estructuración de programas de alimentación escolar
Diogo Thimoteo da Cunha (1) Raquel Braz Assunção Botelho (2) Rafaela Ribeiro de Brito (3) Lívia de Lacerda de Oliveira Pineli (2) Elke Stedefeldt (4)

Grupo de Estudos em Qualidade dos Alimentos - GeQual. ciências. Universidade Federal de São Paulo Santos, SP. Brasil. (2) Departamento de Nutrição. Faculdade de Ciências da Saúde. Universidade de Brasília. Brasília, DF. Brasil. (3)Centro Colaborador em Alimentação e Nutrição Escolar - CECANE. Universidade Federal de São Paulo. Santos, SP. Brasil (4) Grupo de Estudos em Qualidade dos Alimentos - GeQual. Departamento de Gestão e Cuidado em Saúde. Universidade Federal de São Paulo. Santos, SP. Brasil

Dirigir la correspondencia a: Elke Stedefeldt Av. Ana Costa, 178. CEP: $11060-000$ Santos/SP-Brasil

Telefone: +5501338783816 -mail: elke_nutri@hotmail.com

Este trabajo fue recibido el 24 de Junio de 2013 y aceptado para ser publicado el 1 de Noviembre de 2013. en varios países de América Latina, Caribe y África, como: Guatemala, Bolivia, Nicaragua, Haití, Angola, Guinea-Bissau, Cabo-Verde, Santo Tomé y Príncipe, entre otros (3).

El desarrollo y la mejoría de los materiales que se utilizarán en el programa de control de calidad no sólo pueden beneficiar a los escolares brasileños, sino también ayudar a los países que cooperan con Brasil.

El Fondo Nacional para el Desarrollo de la Educación (FNDE) responsable por el PNAE en los últimos años ha invertido en iniciativas para fortalecer la política de seguridad alimentaria y nutricional en las escuelas. Una de estas iniciativas es la creación de Centros Colaboradores en Alimentación y Nutrición Escolar (CECANE). Vinculados a Universidades Federales, los CECANES actúan directamente sobre el desarrollo de la investigación, monitoreo, educación continua de todos los involucrados en el PNAE y asesoramiento a las solicitudes de FNDE $(4,5)$

En 2009, el FNDE tuvo otra iniciativa mediante la publicación de la Resolución №38, que establece disposiciones 
de aplicación del PNAE, e incluye como uno de los procedimientos, las pruebas de aceptación para controlar la calidad de los alimentos servidos a los estudiantes. Estas pruebas deben realizarse cada vez que hay en el menú: la introducción de alimentos atípicos a los hábitos alimentarios locales, otros cambios innovadores en lo que respecta a la preparación y también para evaluar la aceptabilidad de los menús practicados a menudo (6).

Evaluar la aceptación y preferencia de un alimento por el estudiante, de acuerdo con Calil y Aguiar (7) es un factor importante en la determinación de la calidad del servicio prestado por las escuelas en relación con el suministro de comidas escolares.

Cabe señalar que cuando se trata de la preferencia de alimentos y la aceptación por parte de las personas, denota una relación entre los dos, pero no equivalentes. La selección incluye una elección de un producto u otro, es decir, no está necesariamente vinculada a la aceptación o a una actitud positiva hacia ella. Por otro lado, la aceptación es una experiencia hedonista que se caracteriza por una actitud positiva permanente hacia el objeto en cuestión, por lo tanto, visto como una prueba afectiva (8)

Al evaluar la aceptabilidad de los menús de las comidas escolares, se puede utilizar varios métodos, además de la escala hedónica. Entre esa lista son los tradicionales los que relacionan y verifican la cantidad de sobras y residuos de comida. Las técnicas utilizadas incluyen una estimación visual de los residuos, medidas de sobras añadidas, la evaluación de residuos, entre otros. Dependiendo de la característica de cada escuela, cada método puede ser utilizado con el fin de ser una aplicación más rápida y sencilla, así como satisfacer mejor las necesidades del local $(7,9)$.

Con respecto a la escala hedónica, Lawless y Heymann (10) se refieren a ella como el método afectivo más utilizado en las pruebas sensoriales, debido a sus resultados informativos. Fue desarrollado en 1957 como una manera de medir la aceptación de un producto, y en los últimos años, se ha adaptado de acuerdo con el público objetivo.

La práctica de las pruebas de aceptabilidad recomendadas ha demostrado que se hacen varios ajustes, a menudo inducidas por las interpretaciones personales. Se cree que la falta de uniformidad en la aplicación y interpretación de las pruebas ha contribuido a distorsiones en la elección de los productos comprados. Las distorsiones y interpretaciones erróneas pueden interferir en la satisfacción de las necesidades nutricionales de los escolares.

Por lo tanto, este trabajo se justifica por la necesidad de normalizar y definir los métodos que se utilizan en la vida diaria de los responsables de las comidas escolares, que trabajan en diferentes esferas de gobierno (municipal, estatal y federal), así como fomentar su uso en otros países.

El objetivo de este estudio es validar los métodos para verificar la aceptabilidad de las comidas escolares.

\section{SUJETOS Y MÉTODO}

Planificación experimental

Se efectuó un estudio de tipo transversal, de carácter exploratorio con el método comparativo. Se seleccionaron aleatoriamente tres escuelas municipales de la ciudad de Santos (Sao Paulo - Brasil). Los criterios de inclusión en la encuesta fueron los siguientes: ser estudiante del segundo al quinto año de las escuelas, de ambos sexos, con edades comprendidas entre siete y diez años.

Se utilizó el método de muestreo aleatorio simple elegido en los principales estudios similares $(11,12)$. El tamaño de la muestra, basándose en las directrices Batlett y col. (13), fue calculado por el método de Cochran (14), asumiéndose el nivel alfa (error de tipo I) de 5\%, margen de error del 3\%, y estratificación en tres regiones de la ciudad (15). En esta perspectiva, se consideraron alrededor de 120 estudiantes por región, por un total de 360 estudiantes al principio, número suficiente, teniendo en cuenta las ausencias y rechazos.

Se decidió por la estratificación por regiones de la municipalidad a considerar las diferentes realidades socioeconómicas y demográficas, que son: la región central, las colinas y de la zona noroeste.

Después fue sorteada una escuela en cada una de las regiones del municipio y en cada escuela, cuatro clases para participar en la investigación. El número de clases fue establecido porque el promedio era de 30 estudiantes por clase.

\section{Procedimientos}

Para la validación de los métodos se utilizaron la comparación entre los siguientes métodos: tarjetas lúdicas (figura 1), escala hedónica facial mixta de cinco puntos (figura 2) y análisis de los residuos, estos dos últimos ya preconizados por el FNDE para la evaluación de la aceptabilidad (6).

El uso de las tarjetas lúdicas es un nuevo método no validado, pero ya es utilizado por nutricionistas de comidas escolares. En él, el estudiante decide el grado que le gustó o no le gustó el menú ofrecido a través de un conjunto de fichas individuales con las mismas "caras" que se utiliza en la escala hedónica.

Cada escolar recibió un conjunto de cinco fichas con las siguientes entradas correspondientes a cada "cara": odié, no me gustó, indiferente, me gustó y me encantó. La tarjeta lúdica seleccionada, referente a la opinión del escolar, fue colocada en una urna como un sistema de votación. Los datos fueron registrados en una hoja de cálculo de control.

Mediante el uso de fichas no desechables, este método destaca porque es económicamente viable, con la perspectiva de la sostenibilidad del medio ambiente, evitando el gasto en papel y grabados. La figura 1 muestra las fichas utilizadas en el método de tarjeta lúdica.

La prueba de escala hedónica utilizada en la investigación fue descrito por Vieira (12) y Lawless y Heymann (10). Se eligió la escala hedónica facial mixta con cinco "caras" que representan las clasificaciones: odié, no me gustó, indiferente, me gustó y me encantó y el número correspondiente de 1 a 5 . El estudiante llenó la ficha indicando el grado que le gustó o que le disgustó el menú servido. La figura 2 muestra la ficha utilizada para la prueba de escala hedónica facial mixta.

El análisis de los residuos comprendió el método descrito por Abreu y col. (16), calculando el porcentaje de rechazo y la aceptación que se basa en la obtención de los pesos relacionados con la comida rechazada y la comida distribuida, como las ecuaciones presentadas:

Ec1: Porcentaje de rechazo $=($ Peso de la comida rechazada $\times 100)$

$$
\text { Peso de la comida distribuida }
$$

\section{Ec2: Porcentaje de aceptación $=100$ - Porcentaje de rechazo}

Se considera, comida rechazada el peso de los alimentos que han sido rechazados y dejados en el plato y la comida distribuida, la resta entre el peso de la comida producida y la sobra que no ha sido servida a los estudiantes.

Los procedimientos de prueba se produjo en tres escuelas en el siguiente orden: primera evaluación se aplicó por medio 
de la escala hedónica facial mixta junto con el análisis de los desechos en las tres escuelas.

Una semana después de esta aplicación se ha realizado la prueba de aceptación utilizando la tarjeta lúdica y otra vez. Vale la pena señalar que el menú era el mismo que del primer momento.

Para reducir el sesgo en el acuerdo entre los métodos, en las escuelas I y III la prueba se aplicó a un menú con gran aceptación y en la escuela II, con baja aceptabilidad por los escolares. Y para minimizar el sesgo de la tasa de aceptación, las comidas fueron preparadas por el mismo responsable en los dos momentos de recolección de datos. El profesional tenía instrucciones de no cambiar la cantidad y / o el tipo de ingredientes utilizados.

Durante la prueba de análisis de los residuos, cada plato recibió un código que permitió la identificación de los datos. Cada plato fue pesado en tres ocasiones: cuando estaba limpio, con la comida racionada por la cocinera y con los desechos. Es importante mencionar que los platos se pesaron vacíos por no tener un peso regular.

Para el pesaje se utilizó una balanza de precisión digital con capacidad de $5 \mathrm{Kg}$ y escala de $1 \mathrm{~g}$ de la marca Plenna®,

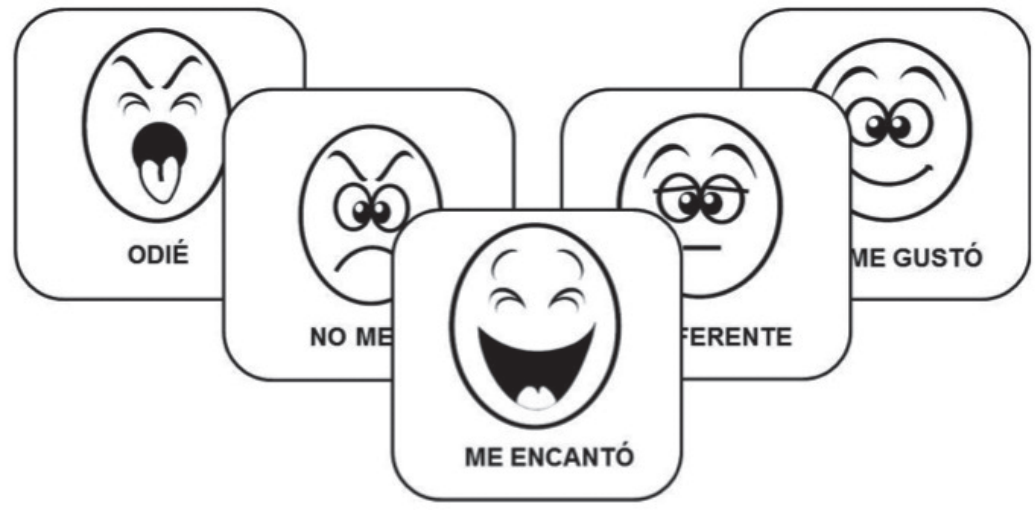

FIGURA 2

Ficha de escala hedónica facial mixta de cinco puntos

Prueba de aceptación de la alimentación escolar

Nombre:

Año:

Fecha:

Señala la carita que más representa lo que te pareció el

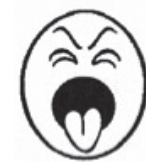

Odié

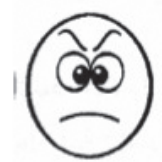

No me gustó

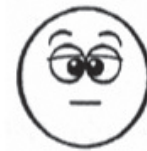

Indiferente

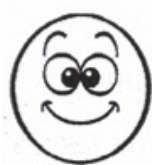

Me gustó

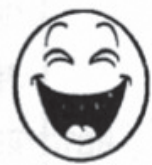

Me encantó

Escribe lo que más te gustó en la preparación:

Escribe lo que menos te gustó en la preparación 
modelo MLA-09100.

La recolección de datos fue realizada por nutricionistas después de la formación específica, incluyendo el tiempo de práctica en la escuela. Se desarrolló un manual de campo que contiene los materiales necesarios para realizar la prueba, así como, el paso a paso de las acciones a realizar.

\section{Análisis}

Los datos fueron procesados y analizados utilizando el software Stata.

Para evaluar el acuerdo entre las diferentes pruebas que utilizó el análisis de coeficiente kappa. Debido a que la evaluación de la aceptabilidad, en un solo menú, ser un indicador multicategórico y ordinal, se realizó una estadística kappa con pesos cuadráticos considerando la clasificación de la aceptación en cinco categorías (17).

La estadística con pesos cuadráticos (PC) se llevó a cabo sólo cuando se compara la escala hedónica facial (EHFPC) y el método de tarjeta lúdica (TLPC) al considerar las cinco categorías de evaluación (lo odié, no me gustó, indiferente, me gustó y me encantó) una vez que los datos del análisis de los desechos siguió siendo caracterizado como una variable continua.

Para otros análisis estadísticos las variables fueron dicotómicas. Los valores 1, 2 y 3 de la escala hedónica y de la tarjeta lúdica (métodos afectivos) se agruparon para categorizar esa región de la escala como una zona de rechazo / indiferencia y la agrupación de los valores 4 y 5 , respecto a los términos hedónicos "me gustó" y "me encantó", corresponden a la región de aceptación de la escala. Lo mismo se hizo con el grado de aceptación calculado por el análisis de los residuos, teniendo en cuenta por debajo del 85\% el área de rechazo / indiferencia y superior o igual al 85\% el área de aceptación.

Los agrupamientos permitieron la elaboración de una tabla de contingencia ( $2 \times 2)$ teniendo en cuenta las siguientes intersecciones: TL x EHF; TL x ARTL; EHF x AREH e; AREH $x$ ARTL. Se entiende por EHF: escala hedónica facial; TL: tarjeta lúdica; $A R E H$ : análisis de residuo realizada conjuntamente con la escala hedónica facial y ARTL: análisis de residuo realizado junto con la tarjeta lúdica. De estos cruces se calculó el coeficiente kappa y el error estándar.

En todas las pruebas se consideró significativo valor de $p<0,05$

\section{Consideraciones éticas}

Los padres o tutores de los escolares firmaron un Formulario de Consentimiento Informado según la Resolución CNS 196/96. El proyecto fue aprobado por el Comité de Ética e investigación de la Universidad Federal de São Paulo con el número CEP 0307/08.

\section{RESULTADOS}

En la investigación participaron 354 escolares de ambos sexos, de tres escuelas, una de cada región de la ciudad de Santos, SP, seis escolares no estaban presentes en dos días de evaluación. Justifica la diferencia en la muestra debido a la falta de estudiantes en cualquiera de los días de la prueba, hecho que no perjudica el estudio.

La figura 3 muestra los valores de acuerdo entre las pruebas evaluadas. La comparación de los datos relativos a la aplicación de la escala hedónica facial y tarjeta lúdica utilizando peso cuadrático con cinco categorías (x TLPC EHFPC) fue el que mostró el mayor acuerdo (92,87\%).

Para las pruebas incluyendo las variables dicotómicas, se

Concordancia y no concordancia entre los métodos de aceptación: tarjeta lúdica (con un peso cuadrático y dicotómico) escala hedónica facial (con un peso cuadrático y dicotómico) y el análisis de residuos.

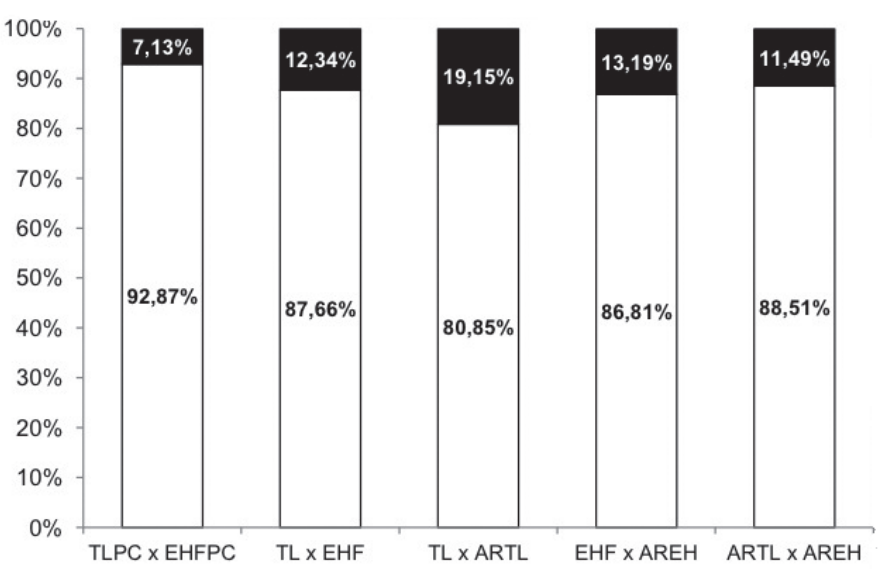

口Concordanica $\mathbf{E}$ No Concordancia

TLPC = tarjeta lúdica con peso cuadrático; $E H F P C=$ escala hedónica facial mixta con peso cuadrático; TL= tarjeta lúdica; EHF= escala hedónica facial mixta;

$\mathrm{AREH}=$ Análisis de residuos realizada en conjunto con la escala hedónica; $\mathrm{ARTL}=$ análisis de residuos realizada en conjunto con la tarjeta lúdica. 
puede ver que la tarjeta lúdica presentó alta concordancia con los dos métodos evaluados: escala hedónica facial $(87,66 \%)$ y el análisis de los residuos (80,85\%), así que los valores esperados fueron de 81,89 y $73,56 \%$, respectivamente.

La concordancia entre la escala hedónica facial con el análisis de los residuos (EHF x AREH) y entre los dos análisis de residuos (uno realizado en el momento de la evaluación con la tarjeta lúdica y otro en el momento de la evaluación con la escala hedónica facial - ARTL x AREH) también mostró una considerable concordancia (figura 3 ).

La tabla 1 muestra los valores de los coeficientes kappa entre los cruces realizados. Los valores observados mostraron que la escala hedónica facial mixta presentó un acuerdo razonable con la tarjeta lúdica utilizando el coeficiente kappa con pesos cuadráticos (kappa con pesos cuadráticos 0,3846). Lo mismo ocurrió con el cruce de las variables dicotómicas, donde la tarjeta lúdica presentó kappa de 0,3186 en respecto a la escala hedónica facial y kappa de 0,2758 en respecto con el análisis de los residuos.

La escala hedónica facial mixta también presentó una concordancia razonable con el método de análisis de los residuos con kappa de 0,2414. La relación entre las dos evaluaciones del análisis de los residuos presentó una buena concordancia con kappa de 0,5566. Todos los análisis presentaron un $p<0,0001$.
La tabla 2 muestra los valores observados en las pruebas de aceptabilidad utilizando los métodos aceptables de tarjeta lúdica, escala hedónica facial y análisis de los residuos.

Con la excepción de la prueba realizada con la tarjeta lúdica en la escuela II, en todas las otras pruebas el índice de aceptabilidad se observó por encima del $85 \%$. Para el análisis de residuos, la escuela II en la segunda prueba mostró un valor bajo del punto de corte recomendado.

\section{DISCUSIÓN}

Los resultados mostraron una concordancia entre los métodos de la escala hedónica facial mixta y tarjeta lúdica con el análisis de residuos, lo que indica que ambos reflejan el nivel de aceptabilidad. La tarjeta lúdica se presentó como un nuevo método, fácil de usar y la aplicación de las pruebas de aceptación para el PNAE ecológicamente sostenible, sin impedimentos a su uso como una prueba de aceptabilidad de los alimentos por los alumnos en el grupo de edad estudiado.

La evaluación de la aceptabilidad de las comidas escolares es aún un tema nuevo, con pocos estudios publicados, a pesar de su importancia como herramienta para el control de calidad. No se encontraron en ambas bases de datos, estudios nacionales e internacionales sobre la validación de métodos afectivos para evaluar la aceptabilidad del menú en el contexto escolar.

\section{TABLA 1}

Valores de los coeficientes Kappa de los cruces entre las pruebas de aceptación: tarjeta lúdica, escala hedónica facial y el análisis de residuo.

\begin{tabular}{|c|c|c|c|c|c|}
\hline \multirow[b]{2}{*}{$\begin{array}{l}\text { Cruce entre las pruebas } \\
\text { de aceptación }\end{array}$} & \multirow[b]{2}{*}{ Kappa } & \multicolumn{4}{|c|}{ Intervalo de confianza 95\% } \\
\hline & & $\begin{array}{c}\text { Error } \\
\text { Estándar }\end{array}$ & $\begin{array}{l}\text { Límite } \\
\text { inferior }\end{array}$ & $\begin{array}{l}\text { Límite } \\
\text { superior }\end{array}$ & $\mathrm{p}$ \\
\hline TLPC $\times$ EHFPC & $0,3846^{*}$ & 0,0625 & 0,224 & 0,534 & $<0,0001$ \\
\hline$T L \times E H F$ & 0,3186 & 0,0630 & 0,125 & 0,492 & $<0,0001$ \\
\hline$T L \times A R T L$ & 0,2758 & 0,0633 & 0,154 & 0,448 & $<0,0001$ \\
\hline EHF $\times$ AREH & 0,2414 & 0,0636 & 0,051 & 0,362 & $<0,0001$ \\
\hline ARTL $\times$ AREH & 0,5566 & 0,0626 & 0,442 & 0,736 & $<0,0001$ \\
\hline
\end{tabular}

TABLA 2

Porcentaje de aceptación según escala hedónica facial, tarjeta lúdica y análisis de residuo en las escuelas evaluados.

\begin{tabular}{|c|c|c|c|c|c|c|}
\hline \multirow[b]{2}{*}{$\begin{array}{l}\text { Término hedónico/ } \\
\text { aceptación }\end{array}$} & \multicolumn{3}{|c|}{ Escala Hedónica Facial (\%) } & \multicolumn{3}{|c|}{ Tarjeta Lúdica (\%) } \\
\hline & $\begin{array}{l}\text { Escuela I } \\
(n=104)\end{array}$ & $\begin{array}{l}\text { Escuela II } \\
(n=111)\end{array}$ & $\begin{array}{l}\text { Escuela III } \\
\qquad(n=73)\end{array}$ & $\begin{array}{l}\text { Escuela I } \\
(n=90)\end{array}$ & $\begin{array}{l}\text { Escuela II } \\
(n=102)\end{array}$ & $\begin{array}{l}\text { Escuela III } \\
\quad(n=89)\end{array}$ \\
\hline 5 - Me encantó & 93 & 79,27 & 56,94 & 90 & 69,6 & 73,03 \\
\hline 4 - Me gustó & 3 & 9 & 30,1 & 5,55 & 4,90 & 16,85 \\
\hline 3 - Indiferente & 0,96 & 4,50 & 9,58 & 1,11 & 9,80 & 5,61 \\
\hline 2 -No me gustó & 0,96 & 1,80 & 1,36 & 0 & 4,90 & 1,12 \\
\hline 1 - Odié & 0,96 & 5,40 & 2,73 & 3,33 & 10,78 & 3,37 \\
\hline $\begin{array}{l}\text { Aceptación por método } \\
\text { afectivo (\%) }\end{array}$ & 96 & 88,27 & 87,04 & 95,55 & 74,5 & 89,88 \\
\hline $\begin{array}{l}\text { Aceptación por análisis } \\
\text { de residuo (\%) }\end{array}$ & 98,4 & 86,32 & 94,76 & 97,4 & 75,82 & 97,76 \\
\hline
\end{tabular}


Stone y Sidel (18) consideran la validez de los métodos sensoriales como el grado en que los resultados de determinada prueba son consistentes con los hechos. En este contexto, este estudio muestra la concordancia de las prueba (tarjeta lúdica y escala hedónica facial) con el hecho (análisis de residuo). Esta relación muestra la concordancia entre dos métodos de distintas naturalezas, siendo dos afectivos y uno cuantitativo.

También se observó una buena concordancia entre el análisis de los residuos realizado en conjunto con la tarjeta lúdica y la escala hedónica facial mixta. Este hecho presenta una buena reproducibilidad de los análisis de los residuos.

Domene y cols (19) encontraron una buena concordancia de una escala hedónica de tres puntos con el análisis de los residuos aplicado a niños. Los resultados son similares y complementarios para el presente estudio. Domene y cols han trabajado con niños de 4 a 6 años y la escala de 3 puntos y en este estudio se utilizó una escala hedónica de 5 puntos con niños de 7 a 10 años.

La creación y validación de métodos tiene como objetivo la evaluación de los menús de los comedores escolares y está en línea con las nuevas políticas y directrices del PNAE con la publicación de la Ley 11.947 (20) y Resolución № 38 (6), que establece la aceptación como forma de control de calidad de las comidas escolares.

Al finalizar este estudio, el grupo de trabajo designado por FNDE preparó el manual de aplicación para las pruebas de aceptación $y$, mediante los resultados positivos, el mismo recomienda el uso de tarjetas lúdicas y escala hedónica facial mixta de cinco puntos para evaluar la aceptabilidad (21).

El análisis estadístico utilizado en el estudio (coeficiente kappa) se utiliza para verificar la concordancia de escalas nominales que determinan la presencia o ausencia de una determinada condición, en este caso, la aceptación y la no aceptación (22). Raghavendra y Antony (23) también indican la importancia en el uso de la estadística kappa en la evaluación de concordancia y validación de métodos. Menciona que el uso de coeficientes de correlación, como Pearson y Spearman en los estudios de validación puede llevar a conclusiones engañosas e incorrectas.

El coeficiente kappa se utiliza con frecuencia en los estudios para la validación de instrumentos de investigación en alimentación y nutrición, especialmente en la validación de Cuestionarios de Frecuencia Alimentaria $(24,25)$.

Los valores más bajos de aceptación se observaron en la escuela II, lo que se esperaba, ya que es una preparación de baja aceptación.

\section{CONCLUSIÓN}

Se validó el uso de tarjetas lúdicas y demostró la concordancia de la escala hedónica facial mixta de cinco puntos y el análisis de los desechos. Los tres métodos para aplicación de la prueba de aceptabilidad están aptos para evaluar la aceptabilidad.

La creación de métodos para las pruebas de aceptación en el contexto escolar se considera un reto debido a sus habilidades cognitivas, participación de la población, la formación, las herramientas para ser utilizadas por los investigadores y los recursos financieros, por lo que los resultados obtenidos en esta investigación son de considerable relevancia.

El uso de métodos simple, ecológicamente sostenibles no sólo facilita la verificación de la calidad y aceptabilidad de las comidas escolares, sino que también, puede promover la participación de los directivos y responsables de la escuela.
Es esencial que estos actores comprendan y experimenten el desafío y lo entiendan, pues son facilitadores en el proceso de mejora de los alimentos servidos en su escuela.

Se recomienda que los métodos validados en un entorno escolar sean aplicados en las diferentes esferas de gobierno, sino también en otros países.

\section{RESUMEN}

El objetivo de este estudio fue validar métodos para evaluar la aceptabilidad de la alimentación escolar. El presente estudio incluyó a 354 estudiantes de segundo año a quinto de las escuelas públicas de la ciudad de Santos - São Paulo Brasil. Para la validación se utilizó de la comparación de tres métodos: Mezcla de escala hedónica facial de cinco puntos, tarjeta lúdica y análisis de residuos, siendo este último el método de referencia. Se utilizó el índice kappa y kappa cuadrática para evaluar el acuerdo entre los métodos. Se estimó un acuerdo razonable entre el análisis de los residuos con los dos métodos afectivos: escala hedónica facial mixta (kappa = 0,2414 ) y tarjeta lúdica (kappa $=0,2758$ ) todos con $p<0,001$. En comparación con la escala hedónica mixta y la tarjeta lúdica, se observó kappa cuadrática de 0,3846. Estos datos muestran la correlación entre los métodos, pero es el evaluador quien elige el uso más apropiado. Se recomienda que la aplicación de los métodos validados en el contexto escolar sea estudiado en las distintas esferas de gobierno y así como en otros países.

Palabras clave: Alimentación escolar; estudios de validación; estudiantes; alimentación.

Agradecimientos: El equipo agradece al Fondo Nacional para el Desarrollo de la Educación (FNDE) por el apoyo a la investigación. Al personal del Centro Colaborador en Alimentación y Nutrición Escolar por la asistencia en la realización del trabajo e las maestras Sueli Silva y Vanessa Capriles por la ayuda en la revisión del manuscrito.

\section{BIBLIOGRAFÍA}

1. Sturion GL, Silva MV, Ometto AH, Furtoso CM, Pipitone, MP. Fatores condicionantes da adesão ao Programa de Alimentação Escolar no Brasil. Rev Nutr; 2005,18(2):167-81.

2. Fundo Nacional de Desenvolvimento da Educação (BR) [site de internet] Ministério da Educação e Cultura - MEC, Brasilia (DF) 2007 [Acesso em 07 Fev 2008]. Disponível em: http://www.fnde.gov.br/home/index.jsp?arquivo=/ alimentacao_esco lar/alimentacao_esc.html.

3. Organization of American States. Cúpula das Américas Relatório Brasil. 2008. Disponível em <http://www.summit-americas.org/Nat\%20Reports/Bra_NR_2008_Pt.pdf> Acesso em: 13 dez 2011.

4. Gaglianone CP, Devincenzi UM, Batista, SS, Stedefeldt E, Castro PM. Centro Colaborador em Alimentação e Nutrição Escolar - CECANE UNIFESP: Finalidade e projetos. Rev Assoc Bras Nutr. 2009; 2(2):49:53.

5. Brasil. Ministério da Educação, Ministério da Saúde. Portaria Interministerial 1.010 de 8 de maio de 2006. Institui as diretrizes para a Promoção da Alimentação Saudável nas Escolas de educação infantil, fundamental e nível médio das redes públicas e privadas, em âmbito nacional. Brasília (DF); 2006.

6. Brasil. Resolução/FNDE/CD/N 38, de 16 de julho de 2009. Estabelece as normas para a execução do Programa Nacional de Alimentação Escolar - PNAE. Brasília (DF); 2009.

7. Calil R; Aguiar J. Nutrição e administração nos serviços de alimentação escolar. São Paulo: Marco Markovitchi, 1999. 
8. Stone $H$, Sidel JL. Affective tests. Sensory Evaluation Practices, 3ed Elsevier Academic Press. p.247-277, 1999.

9. Costell E, Durán L. El análisis sensorial en el control de calidad de los alimentos. I. Introducción. Rev Agroquim Aliment. 1981;21(1):1-10.

10. Lawless HT; Heymann H. Sensory evaluation of food: principles and practices. Acceptance and preference testing. Maryland: Aspen Publishers. p.430-479, 1999.

11. Schutz, HG. A food action rating scale for measuring food acceptance. J. Food Science. 1965;30(2):365-374.

12. Vieira IC. Métodos de Aceitação em Merenda Escolar [dissertação de mestrado]. Campinas; Universidade Estadual de Campinas. 1981.

13. Bartlett JE, Kotrlik, JW, Higgins C. Organizational research: Determining appropriate sample size for survey research. Inform Tech Learn Perform J. 2001;19(1) 43-50.

14. Cochran WG. Sampling Techniques. $3^{a}$ ed. New York: John Wiley \&Sons, 1977.

15. Krejcie RV, Morgan DE. Determining sample size for research activities. Educational and Psychological Measurement. p.607-610; 1970.

16. Abreu ES, Spinelli MN, Zanardi AP. Gestão de Unidades de Alimentação e Nutrição: um modo de fazer. São Paulo: Editora Metha Ltda, p.110-113, 2003.

17. Altman DR. Practical Statistics for Medical Research. N.York: Chapman \& Hall/CRC, p.200-215, 1999.

18. Stone $H$, Sidel JL. Test strategy and design of experiments.
Sensory Evaluation Practices, Elsevier Academic Press, p.99-137, 2004.

19. Domene SMA, Zapico J, Taddei JAAC. Adaptación de la escala hedónica facial para medir preferencias alimentarias de alumnos de pre-escolar. Rev Chil Nutr. 2008; 35(1):38-42.

20. Brasil. Lei 11.947 de 16 de Junho de 2009, Dispõe sobre o atendimento da alimentação escolar e do Programa Dinheiro Direto na Escola aos alunos da educação básica. Brasilia, DF, 2009.

21. Cecane Unifesp. Manual para aplicação dos testes de aceitabilidade no Programa Nacional de Alimentação Escolar - PNAE. 2010. Disponivel em <http://www. fnde.gov.br/index.php/arq-alimentacao-escolar/5096manualaplicacaotestesdeaceitabilidadepnae/download> Acesso em: 20 de janeiro de 2011.

22. Sim J, Wright C. The Kappa Statistic in Reliability Studies Use, Interpretation, and Sample Size Requirements. Phys Therapy. 2005; 85(3) 257-26.

23. Raghavendra D, Antony G. Categorical Data Analysis. Applied Clinical Trials. 2011; 20(5) 46-51.

24. Penkilo M, George GC, Hoelscher DM. the School-based Nutrition Monitoring Questionnaire among Fourth-grade Students in Texas. J Nutr Educ Behav. 2008;40(1):20-7.

25. Vähätalo L, Bärlund S, Hannila M, Uusitalo U, Pigg HM, Salonen $M$, et al Relative validity of a dietary interview for assessing infant diet and compliance in a dietary intervention trial. Mat Chil Nutr. 2006; 2:181-7. 